Annuaire du Collège de France 2017-2018

\title{
Physique statistique
}

\section{Bernard Derrida}

\section{OpenEdition}

Journals

Édition électronique

URL : https://journals.openedition.org/annuaire-cdf/15478

DOI : 10.4000/annuaire-cdf. 15478

ISBN : 978-2-7226-0572-5

ISSN : 2109-9227

Éditeur

Collège de France

Édition imprimée

Date de publication : 30 décembre 2020

Pagination : $51-56$

ISBN : 978-2-7226-0516-9

ISSN : 0069-5580

\section{Référence électronique}

Bernard Derrida, « Physique statistique », L'annuaire du Collège de France [En ligne], 118 | 2020, mis en

ligne le 01 avril 2021, consulté le 22 août 2022. URL : http://journals.openedition.org/annuaire-cdf/ 15478 ; DOI : https://doi.org/10.4000/annuaire-cdf.15478 


\title{
PHYSIQUE STATISTIQUE
}

\author{
Bernard DERRIDA \\ Membre de l'Institut (Académie des sciences), \\ professeur au Collège de France
}

Mots-clés : physique statistique, équation KPZ

La série de cours et séminaires «Désordre, croissance et exclusion » est disponible en audio et en vidéo, sur le site internet du Collège de France (https://www.collegede-france.fr/site/bernard-derrida/course-2017-2018.htm).

\section{ENSEIGNEMENT}

\section{COURS ET SÉMINAIRES - DÉSORDRE, CROISSANCE ET EXCLUSION}

Des systèmes aussi différents que des problèmes de croissance de colonies de bactéries, de combustion de papier, de déplacement de parois magnétiques ou de trafic routier relèvent tous d'une même théorie. Cette théorie au cœur de laquelle se trouve l'équation KPZ (Kardar Parisi Zhang) qui date de 1986 a connu au cours des deux dernières décennies des avancées spectaculaires. Après avoir présenté quelques résultats d'expériences et de simulations numériques de systèmes pouvant être décrits par l'équation KPZ, le but du cours a été d'introduire les principaux modèles (modèles d'exclusion, polymères en présence de désordre, modèles de sédimentation) qui relèvent de cette théorie et d'exposer les principales approches théoriques permettant de les étudier.

\section{Cours du 15 janvier 2018}

Le premier cours a commencé par introduire certains des modèles les plus simples, comme le modèle d'Eden ou le modèle déposition balistique, qui décrivent la croissance d'un milieu stable vers un milieu instable. Ces modèles très faciles à 
simuler sur ordinateur permettent d'observer l'évolution des fluctuations de la frontière qui sépare le milieu stable du milieu instable. La distribution de ces fluctuations obéit à des lois d'échelle indépendantes du modèle précis considéré comme la distribution de Tracy Widom découverte dans les années 1990 dans un tout autre contexte, celui de la théorie des matrices aléatoires. Des approches théoriques et numériques de Michael Prähofer et Herbert Spohn ont en effet permis de prédire les lois statistiques universelles de ces fluctuations en dimension $1+1$ et ces lois ont pu être observées récemment dans des expériences sur des cristaux liquides par Kazumasa A. Takeushi et Masaki Sano. Ce premier cours s'est poursuivi en montrant comment relier les exposants qui régissent ces lois universelles et en introduisant l'équation KPZ. Il s'est terminé en expliquant comment la dépendance directionnelle de la vitesse de croissance permet de prédire la forme macroscopique des domaines de croissance.

\section{Séminaire 1 - Within and beyond the realm of $K P Z$}

\section{Tim Halpin-Healy (Columbia University, New York)}

Ce séminaire a fait une revue des principales avancées passées sur l'équation KPZ et expliqué les raisons du regain d'intérêt récent pour cette énigmatique équation stochastique et non linéaire. Les retombées de ces progrès touchent un grand nombre de domaines, qu'il s'agisse de la croissance hors d'équilibre, des gaz sur réseau, de la théorie des files d'attente, des chemins optimaux dans des paysages aléatoires ou des propriétés statistiques des matrices aléatoires.

\section{Cours du 22 janvier 2018}

Le second cours a permis d'introduire deux autres familles de modèles qui relèvent de la classe d'universalité de l'équation KPZ : les processus d'exclusion asymétriques et les polymères dirigés en milieu aléatoire. Les modèles d'exclusion ont été proposés dans les années 1960 pour décrire le mouvement dirigé d'enzymes le long d'une molécule d'ADN. Ils ont beaucoup été étudiés pour comprendre la limite hydrodynamique qui établit le pont entre le monde microscopique et le monde macroscopique. De plus, ils font partie d'une famille de modèles hors d'équilibre que l'on sait résoudre exactement. En établissant un lien entre les processus d'exclusion et les modèles de croissance, on peut déterminer de manière exacte l'ensemble des exposants pour le problème de croissance en dimension $1+1$. Quelques résultats marquants comme la formule de Gunter M. Schütz (1997) et celle de Kurt Johansson (2000) ont été énoncés, qui ont permis de faire le lien avec la théorie des matrices aléatoires. La suite du cours a été consacrée à la question des polymères dirigés en milieu aléatoire, en montrant en particulier le lien avec le problème de la loi statistique de la plus grande sous-séquence croissante d'une permutation aléatoire.

\section{Séminaire 2 - Random matrix theory and cold atoms}

Satya Majumdar (université de Paris-Sud, Orsay)

Ce séminaire a d'abord donné un bref historique de la théorie des matrices aléatoires et de ses nombreuses applications. Il a ensuite introduit la célèbre distribution de Tracy-Widom qui décrit la probabilité de la plus grande valeur propre 
d'une matrice aléatoire. La deuxième partie a été consacrée à une application de la théorie des matrices aléatoires aux atomes froids en utilisant le lien inattendu entre la position de la particule la plus à droite d'un gaz de fermions confinés, la plus grande valeur propre de matrices aléatoires gaussiennes et la hauteur dans l'équation KPZ.

\section{Cours du 29 janvier 2018}

Le troisième cours a été entièrement consacré à une introduction à la théorie des matrices aléatoires de l'ensemble Gaussian Unitary Ensemble (GUE). Ont d'abord été rappelés les résultats classiques sur la densité et les corrélations des valeurs propres des matrices aléatoires, ainsi que les nombreux domaines de la physique ou des mathématiques où elles sont étudiées (physique nucléaire, chaos quantique, fonction zéta de Riemann, ...). Le cours s'est poursuivi en montrant comment ces résultats découlent d'une représentation de la distribution des valeurs propres en termes d'énergies d'un système de fermions libres, que l'on peut calculer par un traitement semi-classique.

\section{Séminaire 3 - Teaching and learning nonequilibrium statistical physics}

Roberto Livi (université de Florence, Italie)

La physique statistique est un sujet très étudié actuellement, en particulier à cause de son caractère pluridisciplinaire. Le sujet présente cependant certaines difficultés conceptuelles quand il s'agit de l'enseigner, par exemple dans des cours avancés à l'université. Ce séminaire a essayé de mettre en lumière plusieurs de ces difficultés et de montrer comme des approches pédagogiques permettent de les contourner.

\section{Cours du 5 février 2018}

Le quatrième cours a commencé par établir l'équivalence entre l'équation de la chaleur stochastique et l'équation KPZ. En partant du cas des polymères dirigés, il a été montré que l'équation KPZ se réduit, dans la limite des hautes températures, à une équation linéaire, l'équation d'Edwards Wilkinson. Cette équation peut évidemment se résoudre à toute dimension. La suite du développement de haute température permet de montrer que les corrections explosent à basse dimension. La suite du cours a été consacrée à d'autres versions simplifiées de l'équation KPZ : l'équation de Burgers avec ou sans viscosité, qui donne lieu à des ondes de choc, et à la discussion des différentes descriptions possibles de ces ondes de choc à l'échelle miscroscopique.

\section{Séminaire 4 - Le processus d'exclusion, un paradigme de la physique hors d'équilibre}

\section{Kirone Mallick (CEA, Saclay)}

Le processus d'exclusion symétrique est un modèle de particules en interaction, décrivant des phénomènes de transport à basse dimension avec contraintes stériques. Les solutions exactes de ce modèle nous aident à mieux comprendre la physique hors d'équilibre. Ce séminaire a permis d'exposer quelques résultats marquants concernant ce système, de décrire les méthodes mises en œuvre pour les résoudre et de présenter certains développements récents. 


\section{Cours du 12 février 2018}

Le cinquième cours a été essentiellement consacré aux modèles d'exclusion. Après avoir montré comment obtenir le diagramme de phases à partir des solutions de l'équation de Burgers, et le lien des modèles d'exclusion unidimensionnels avec les chaînes de spins quantiques, plusieurs méthodes de solutions exactes ont été expliquées : l'approche matricielle qui permet à la fois de rerouver le diagramme de phase et de comprendre la forme des profils de densité et l'ansatz de Bethe qui est à l'origine de la formule de Schütz présentée lors du deuxième cours.

\section{Séminaire 5 - Applications des processus d'exclusion au trafic routier, piétonnier, et intracellulaire}

\section{Cécile Appert-Rolland (université de Paris-Sud, Orsay)}

Après une brève revue des familles de modèles utilisés pour décrire le trafic routier, piétonnier ou intracellulaire, plusieurs généralisations des processus d'exclusion appliqués à ces systèmes de transport ont été décrits. L'introduction d'un temps de réaction permet de modifier la structure spatio-temporelle de l'écoulement sur une autoroute. La modélisation de piétons a montré l'importance de l'algorithme de mise à jour. Un processus d'exclusion bidimensionnel permet de comprendre la formation spontanée de diagonales au croisement de deux flux perpendiculaires de piétons. Enfin, le séminaire s'est conclu par des questions de transport intracellulaire et la mise en évidence par des simulations numériques de quelques effets contre-intuitifs qui peuvent se produire lorsque des moteurs moléculaires se déplacent le long de réseaux de microtubules.

\section{Cours du 19 février 2018}

Le sixième cours a présenté plusieurs aspects de la théorie des polymères en milieu aléatoire. Ils constituent l'un des systèmes les plus étudiés dans la théorie des systèmes désordonnés. Le cours a commencé par montrer la différence entre moyenne recuite et moyenne gelée. Puis il a été montré que les polymères en milieu aléatoire présentent une transition de phase en dimension supérieure à trois, avec une phase de haute température où le polymère est délocalisé et une phase de basse température où le polymère est piégé. En dimension inférieure ou égale à trois, seule la phase piégée subsiste. Le cours s'est poursuivi en montrant comment la solution du modèle de Lieb Liniger par l'ansatz de Bethe permet de calculer l'énergie libre des polymères dirigés en dimension $1+1$ en utilisant la méthode des répliques.

\section{Séminaire 6 - Chemins en milieu aléatoire et mécanique quantique de bosons en interaction}

\section{Pierre Le Doussal (ENS, Paris)}

Une façon d'aborder la question de l'équation KPZ est de considérer le problèmes de polymères dirigés en milieu aléatoire que l'on peut traiter par la méthode des répliques. En dimension $1+1$ et dans sa version continue dans le temps et dans l'espace, ce problème se réduit à l'étude d'un gaz de bosons en interaction delta, un problème que Lieb et Liniger ont résolu dans les années 1960. La difficulté à surmonter est que, pour trouver la distribution de l'énergie libre des polymères, c'est 
à dire de la hauteur dans l'équation KPZ, par la méthode des répliques, il faut pouvoir donner un sens à un gaz de bosons avec un nombre non entier de particules. En décrivant les aspects techniques utilisés pour résoudre cette difficulté, Pierre Le Doussal a expliqué comment cette approche de l'équation KPZ par la méthode des répliques permet de retrouver la distribution de Tracy Widom.

\section{RECHERCHE}

Mes travaux de recherche au cours de l'année 2017-2018 peuvent se regrouper en cinq thèmes principaux.

\section{La fonction de grande déviation du courant dans des systèmes spatialement périodiques :}

Avec Karel Proesmans de l'université de Hasselt en Belgique, venu passer un semestre postdoctoral au Collège de France, nous avons considéré la fonction de grande déviation d'une particule évoluant selon une dynamique de Langevin sur un cercle. Dans la limite d'un faible bruit, cette fonction de grande déviation présente un point anguleux quand la vitesse s'annule. En utilisant une approche semiclassique, la méthode WKB, nous avons montré comment comprendre la façon dont ce point anguleux est arrondi à l'ordre dominant dans l'amplitude du bruit. Notre approche est très inspirée des calculs d'instantons en mécanique quantique.

\section{Les grandes déviations pour les systèmes hors d'équilibre}

Avec Tridib Sahu du Tata Institute à Bombay, en visite au Collège de France pendant l'année 2017-2018, nous avons poursuivi nos travaux sur les systèmes hors d'équilibre. Dans un premier article déjà terminé et en cours de publication, nous avons considéré le cas de systèmes stochastiques ayant un petit nombre de degrés de liberté et montré comment calculer les mesures stationnaires dont la dynamique est conditionnée par la valeur empirique d'une quantité mesurée sur une longue fenêtre de temps. Nous sommes actuellement en train d'étendre ces résultats au cas de systèmes étendus comportant un nombre macroscopique de degrés de liberté.

\section{Les effets de taille finie et la brisure de symétrie des répliques}

Avec Peter Mottishaw de l'université d'Édimbourg, nous avons poursuivi nos travaux sur les effets de taille finie dans le cas des verres de spins. Nous avons en particulier calculé ces effets de taille finie pour les modèles d'énergies aléatoires (GREM) et cela nous a permis de généraliser nos résultats antérieurs au cas de transitions de verres de spin graduelles.

\section{Désordre et renormalisation}

Avec mon nouvel étudiant Victor Dagard et en étroite collaboration avec Zhan Shi, professeur de probabilités à l'université Pierre et Marie Curie, nous continuons à travailler sur la transition de dépiégeage en présence de désordre. Un de nos progrès récents a été d'obtenir une famille de solutions invariantes d'échelle. Chacune de ces 
solutions correspond à un comportement critique différent et peut être caractérisée par la décroissance de la distribution du désordre à l'infini. Nous sommes actuellement en train de généraliser à cette famille de solutions critiques les résultats obtenus précédemment dans le cas générique.

\section{L'équation F-KPP}

Les travaux sur l'équation Fisher-KPP, en collaboration avec Éric Brunet de l'ENS et Julien Berestycki de l'université d'Oxford, se sont poursuivis, conduisant à une série de résultats exacts sur le développement asymptotique aux temps longs de la position des fronts. Notre progrès principal cette année a été de montrer que les résultats obtenus antérieurement dans le cas d'une équation de type F-KPP linéaire avec saturation restent valables pour un choix général de non-linéarités.

\section{PuBlications}

BERESTYCKI J., BRUNET É. et DERRIDA B., «A new approach to computing the asymptotics of the position of Fisher-KPP fronts », EPL : Europhysics Letters, vol. 122, n 1, 2018, p. 10001, DOI : 10.1209/0295-5075/122/10001 [arXiv:1802.03262, HAL : 01723859].

DERridA B. et MOtTISHAW P., «Finite size corrections to the Parisi overlap function in the GREM », Journal of Statistical Physics, vol. 172, no 2, 2018, p. 592-610, DOI : 10.1007/ s10955-018-1953-9 [arXiv:1710.04611].

BERESTYCKI J., BRUNET É. et DERRIDA B., «Exact solution and precise asymptotics of a Fisher-KPP type front », Journal of Physics A: Mathematical and Theoretical, vol. 51, $\mathrm{n}^{\mathrm{o}} 3$, 2018, 035204, DOI : 10.1088/1751-8121/aa899f [arXiv:1705.08416, HAL : 01723857].

DERRIDA B. et SHI Z., « Slower deviations of the branching Brownian motion and of branching random walks », Journal of Physics A: Mathematical and Theoretical, vol. 50, $\mathrm{n}^{\mathrm{o}}$ 34, 2017, 344001, DOI : 10.1088/1751-8121/aa7f98 [arXiv:1705.02277].

DERRIDA B., «Physique statistique », Annuaire du Collège de France 2015-2016. Résumé des cours et travaux, $\mathrm{n}^{\mathrm{O}} 116,2018$, p. 43-48, DOI : 10.4000/annuaire-cdf.12790 ; en ligne : https:// journals.openedition.org/annuaire-cdf/12790. 